\title{
Rechtliche Grundlagen und Ziele
}

Die Richtlinie 2003/99/EG zur Überwachung von Zoonosen und Zoonoseerregern regelt das gemeinschaftliche Verfahren zur Überwachung von Zoonosen und verpflichtet die Mitgliedstaaten der EU, repräsentative und vergleichbare Daten über das Auftreten von Zoonosen und Zoonoseerregern sowie diesbezüglicher Antibiotikaresistenzen in Lebensmitteln, Futtermitteln und lebenden Tieren zu erfassen, auszuwerten und zu veröffentlichen. Die Daten sollen es ermöglichen, Gefahren zu erkennen und zu beschreiben, Expositionen zu bewerten und die von Zoonosen und Zoonoseerregern ausgehenden Risiken zu beschreiben.

Die Allgemeine Verwaltungsvorschrift über die Erfassung, Auswertung und Veröffentlichung von Daten über das Auftreten von Zoonosen und Zoonoseerregern entlang der Lebensmittelkette (AVV Zoonosen Lebensmittelkette) basiert auf der Richtlinie 2003/99/EG und bildet die Grundlage für das Zoonosen-Monitoring. Die AVV Zoonosen Lebensmittelkette regelt die Vorgehensweise bei der Planung, Koordinierung und Durchführung der Untersuchungen zum Zoonosen-Monitoring und für das anschließende Berichtswesen.

Vorrangig sollen diejenigen Zoonoseerreger überwacht werden, die eine besondere Gefahr für die mensch- liche Gesundheit darstellen. Im Anhang I, Teil A der Richtlinie 2003/99/EG sind die in jedem Mitgliedstaat überwachungspflichtigen Zoonosen und Zoonoseerreger genannt. Weiterhin soll das Überwachungssystem das Erkennen aufkommender und neu aufkommender Zoonoseerreger erleichtern.

Die Überwachung erfolgt auf jenen Stufen der Lebensmittelkette einschließlich der Primärproduktion, die hinsichtlich des jeweiligen Zoonoseerregers am besten dafür geeignet sind. Die Richtlinie 2003/99/EG sieht vor, dass die Überwachung von Resistenzen gegen antimikrobiell wirksame Stoffe neben Zoonoseerregern auch andere Erreger erfasst, wenn diese eine Gefahr für die öffentliche Gesundheit darstellen. Insbesondere müssen die Mitgliedstaaten gewährleisten, dass das Überwachungssystem auf Grundlage des Kommissionsbeschlusses 2013/652/EU zur Überwachung und Meldung von Antibiotikaresistenzen bei zoonotischen und kommensalen Bakterien einschlägige Informationen über eine repräsentative Anzahl von Isolaten von Salmonella spp., Campylobacter spp., kommensalen $E$. coli sowie ESBL/AmpC-bildenden E. coli liefert, die von Rindern, Schweinen und Geflügel sowie den von diesen Tieren gewonnenen Lebensmitteln stammen. 